\section{Acknowledgments}

This work was supported in part by NIH grants NICHD R01-HD054599 and R01HD69045, and by grants from FightSMA, Families of SMA, and the Muscular Dystrophy Association.

Address correspondence to: Kathryn J. Swoboda, Pediatric Motor Disorders Research Program, Department of Neurology, 30 N. 1900 E. SOM3R149, Salt Lake City, Utah 84132, USA. Phone: 801.585.9717; Fax: 801.587.9346; E-mail: swoboda@genetics.utah.edu.

1. Prior TW, et al. Newborn and carrier screening for spinal muscular atrophy. Am J Med Genet Part A. 2010;152A(7):1608-1616

2. Werdnig G. Zwei Frühinfantile hereditäre Fälle von progressiver Muskelatrophie unter dem Bilde der Dystrophie, aber auf neurotischer Grundlage. Arch Psychiat Nervenkr. 1891;22(2):437-480.

3. Hoffman J. Ueber chronische spinale Muskelatrophie im Kindesalter, auf Familiärer Basis. Beutsch Z Nervenheilk. 1893;3(6):427-470.

4. Lefebvre $S$, et al. Identification and characterization of a spinal muscular atrophy-determining gene. Cell. 1995;80(1):155-165.

5 . Wan $\mathrm{L}$, et al. The survival of motor neurons protein determines the capacity for snRNP assembly: biochemical deficiency in spinal muscular atrophy. Mol Cell Biol. 2005;25(13):5543-5551.

6. Feldkotter M, et al. Quantitative analyses of SMN1 and SMN2 based on real-time lightcycler PCR: fast and highly reliable carrier testing and prediction of severity of spinal muscular atrophy. Am J Hum Genet. 2002;70(2):358-368.
7. Mailman MD, et al. Molecular analysis of spinal muscular atrophy and modification of the phenotype by SMN2. Genet Med. 2002;4(1):20-26.

8. Rochette CF, Gilbert N, Simard LR. SMN gene duplication and the emergence of the SMN2 gene occurred in unique hominids: SMN2 is unique to Homo sapiens. Hum Genet. 2001;108(3):255-266.

9. Monani UR, et al. A single nucleotide difference that alters splicing patterns distinguishes the SMA gene SMN1 from the copy gene SMN2. Hum Mol Genet. 1999;8(7):1177-1183.

10. Lorson CL, Hahnen E, Androphy EJ, Wirth B. A single nucleotide in the SMN gene regulates splicing and is responsible for spinal muscular atrophy. Proc Natl Acad Sci U S A. 1998;96(11):6307-6311.

11. Fischer U, Liu Q, Dreyfuss G. The SMN-SIP1 complex has an essential role in spliceosomal snRNP biogenesis. Cell. 1997;90(6):1023-1029.

12. Kolb SJ, Kissel JT. Spinal muscular atrophy: a timely review [published online ahead of print April 11, 2011]. Arch Neurol. doi:10.1001/archneurol.2011.74.

13. Brichta L, et al. Valproic acid increases the SMN2 protein level: a well-known drug as a potential therapy for spinal muscular atrophy. Hum Mol Genet. 2003;12(19):2481-2489.

14. Andreassi C, et al. Phenylbutyrate increases SMN expression in vitro: relevance for treatment of spinal muscular atrophy. Eur J Hum Genet. 2004; 12(1):59-65.

15. Grzeschik SM, et al. Hydroxyurea enhances SMN2 gene expression in spinal muscular atrophy cells. Ann Neurol. 2005;58(2):194-202.

16 . Brichta $L$, et al. In vivo activation of SMN in spinal muscular atrophy carriers and patients treated with valproate. Ann Neurol. 2006;59(6):970-975.

17. Brahe C, et al. Phenylbutyrate increases SMN gene expression in spinal muscular atrophy patients. Eur J Hum Genet. 2005;13(2):256-259.

18. Avila AM, et al. Trichostatin A increases SMN expression and survival in a mouse model of spinal muscular atrophy. J Clin Invest. 2007;117(3):659-671.
19. Foust KD, et al. Rescue of the spinal muscular atrophy phenotype in a mouse model by early postnatal delivery of SMN. Nat Biotechnol. 2010; 28(3):271-274.

20. Dominguez E, et al. Intravenous scAAV9 delivery of a codon-optimized SMN1 sequence rescues SMA mice. Hum Mol Genet. 2011;20(4):681-693.

21. Monani UR, et al. The human centromeric survival motor neuron gene (SMN2) rescues embryonic lethality in $\operatorname{Smn}(-/-)$ mice and results in a mouse with spinal muscular atrophy. Hum Mol Genet. 2000;9(3):333-339.

22. Wirth B, et al. Mildly affected patients with spinal muscular atrophy are partially protected by an increased SMN2 copy number. Hum Genet. 2006;119(4):422-428.

23. Prior TW, et al. Homozygous SMN1 deletions in unaffected family members and modification of the phenotype by SMN2. Am J Med Genet A. 2004;130A(3):307-310.

24. Farooq F, et al. Prolactin increases SMN expression and survival in a mouse model of severe spinal muscular atrophy via the STAT5 pathway. J Clin Invest. 2011;121(8):3042-3050.

25. Lutz CM, et al. Postsymptomatic restoration of SMN rescues the disease phenotype in a mouse model of severe spinal muscular atrophy. J Clin Invest. 2011;121(8):3029-3041.

26. Powe CE, et al. Recombinant human prolactin for the treatment of lactation insufficiency. Clin Endocrinol (Oxf). 2010;73(5):645-653.

27. Kong L, et al. Impaired synaptic vesicle release and immaturity of neuromuscular junctions in spinal muscular atrophy mice. J Neurosci. 2009; 29(3):842-851.

28. Swoboda KJ, et al. Natural history of denervation in SMA: relation to age, SMN2 copy number and function. Ann Neurol. 2005;57(5):704-712.

29. Zerres K, Wirth B, Rudnik-Schoneborn S. Spinal muscular atrophy-clinical and genetic correlations. Neuromuscul Disord. 1997;7(3):202-207.

\title{
Quantity, not quality, of antibody response decreased in the elderly
}

\author{
Bonnie B. Blomberg and Daniela Frasca
}

University of Miami School of Medicine, Miami, Florida, USA.

\begin{abstract}
The burden of disease during seasonal influenza epidemics is felt most keenly among the very young and the elderly. Although vaccination effectively protects children and young adults against infection, it has limited efficacy in elderly individuals. This has been linked to a reduced ability to induce a robust serum antibody response. In this issue of the JCI, Sasaki et al. identify some of the cellular and molecular deficits that underlie the reduced serum antibody response induced by influenza vaccination in elderly individuals. Importantly, they show that it is the quantity of the response, and not its quality, that needs to be improved if we are to enhance the success of influenza vaccination in this vulnerable population.
\end{abstract}

Conflict of interest: The authors have declared that no conflict of interest exists.

Citation for this article: J Clin Invest. 2011;

121(8):2981-2983. doi:10.1172/JCI58406
Influenza epidemics are associated with an estimated 200,000 hospitalizations and 35,000 deaths each year in the United States alone (1). Most of these adversely affected individuals are young children or elderly individuals. Vaccination against influenza has been described to be effective in preventing infection in healthy children and young adults; however, many of the elderly individuals who receive the vaccine still contract the infection and have secondary complications that lead to hospitalization and/or disability $(2,3)$. Despite this, influenza vaccination is still universally recommended for elderly individuals, because it does reduce disease severity, but understanding the mechanism(s) that underlies the limited response of the elderly to influenza vaccines could help direct the development of more effective approaches. 
A
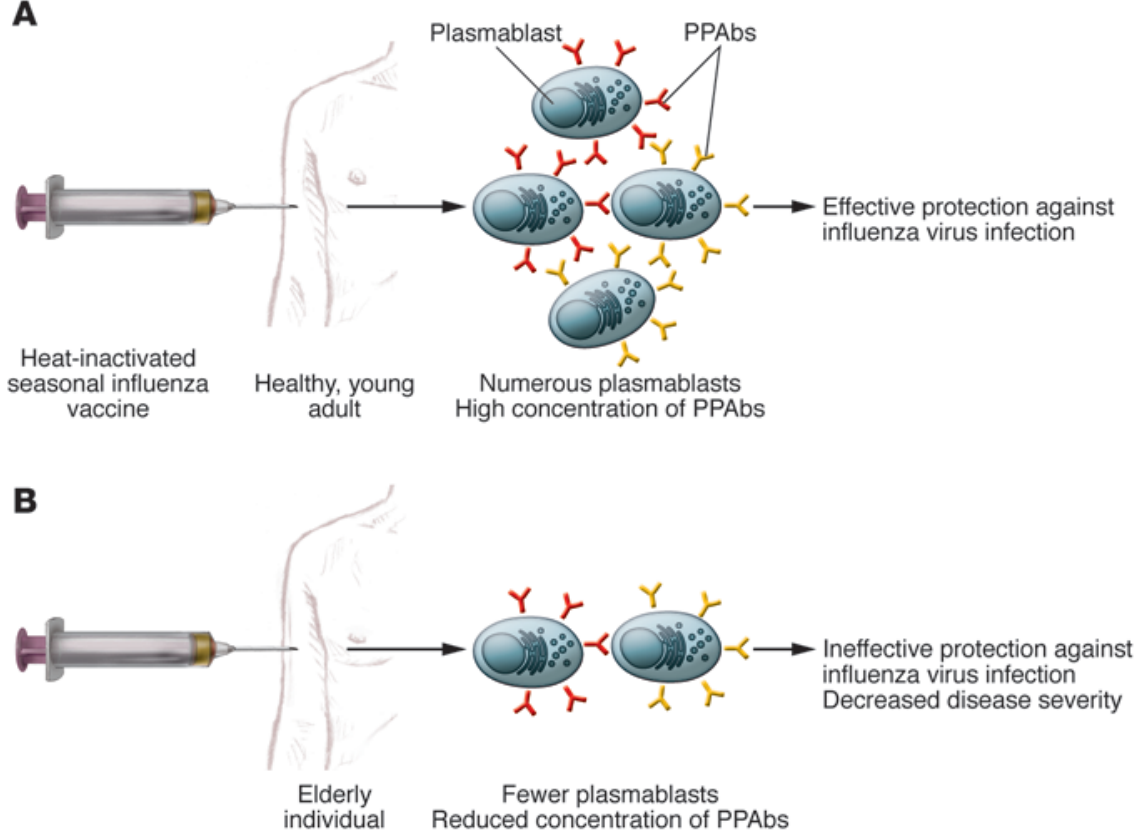

Influenza infection and vaccination

Antibodies play a key role in protecting against infection with the influenza virus, and these are induced upon vaccination with current influenza vaccines, especially in healthy adults $(4,5)$. Vaccination induces a population of circulating antibody-secreting cells (ASCs; also known as plasmablasts), defined as $\mathrm{CD} 19^{+} \mathrm{CD} 20^{\mathrm{lo} /-\mathrm{CD}} 3^{-} \mathrm{CD} 38^{\text {hi }} \mathrm{CD} 27^{\text {hi }}$ cells, which are detected with a peak around seven days after vaccination $(6,7)$. Plasmablasts are short lived, although some of these cells become long lived if rescued in available niches, such as the bone marrow (8). Generation of $\mathrm{T}$ cell responses is also important for eradicating influenza virus infections. In this context, $\mathrm{CD} 4^{+}$helper $\mathrm{T}$ cells activate $\mathrm{B}$ cells to make antibodies, while $\mathrm{CD}^{+}$cytotoxic $\mathrm{T}$ cells destroy virus-infected cells. Current influenza vaccines primarily stimulate an antibody response, and this vaccine-induced antibody response allows time for the host $T$ cell response to develop as well as decreases the initial viral load.

There is substantial evidence that the antibody response induced by influenza vaccines decreases with age (9-11). Most of the studies conducted so far have shown that this correlates with the well-characterized age-dependent decrease in $\mathrm{T}$ cell functions (12-14). However, immune defects with age do not occur in $\mathrm{T}$ cells alone. Recently, agerelated autonomous $B$ cell defects have been described $(15,16)$. These include decreased class switch recombination (CSR), the process by which activated $\mathrm{B}$ cells change the isotype of the antibody that they are producing so that antibodies with the same specificity but different effector function can participate in the immune response; decreased expression of components of the molecular pathway controlling CSR, which include the enzyme activation-induced cytidine deaminase and the transcription factor E47; and decreased levels of switch memory B cells $(15,16)$. These changes completely correlate with the reduced antibody response of elderly individuals to the influenza vaccine (17), and we suggest that they could be used as biomarkers to predict immune/vaccine responsiveness.

Despite agreement that the limited efficacy of vaccination against influenza in the elderly is linked to reduced serum antibody responses, it is not clear whether this is due to decreases in the quantity and/or the quality of the antibodies produced. If it is the quantity, is it due to a decrease in the number of ASCs or in the amount of antibody secreted per cell? The quantity of the serum antibody response is determined by the number of plasmablasts and the amount of antibody secreted by each plasmablast, whereas the quality, or avidity, depends on the affinity of the Igs produced for their cognate antigen. A paper in this issue of the JCI by Sasaki and collaborators addresses these key questions (18). The paper confirms previous studies showing that the serum response, evaluated as fold increase in titer after vaccination, is reduced in elderly individuals and, importantly, extends these find-

\section{Figure 1}

Mechanistic explanation for the inferior antibody response to influenza vaccination in the elderly. The efficacy of influenza vaccination wanes with age. This is linked to a reduced ability to induce a robust serum antibody response. However, whether it is due to decreases in the quantity and/or the quality of the antibodies produced is not clear. In this issue of the $\mathrm{JCl}$, Sasaki et al. answer this question (18), showing that the reduced serum antibody response generated by the elderly after vaccination with inactivated seasonal influenza vaccine is a result of a decrease in the number of responding plasmablasts and hence a decrease in the concentration of PPAbs, rather than a result of a decrease in the quality of the response. Importantly, the amount of antibody secreted was about the same for individual plasmablasts isolated from (A) young and (B) elderly individuals. ings to show that the number of responding plasmablasts and hence plasmablast-derived polyclonal antibodies (PPAbs) is dramatically reduced in elderly individuals compared with that in young individuals (Figure 1). In addition to answering the questions above, Sasaki et al. have efficiently used a system, previously established by Wilson, Ahmed, and colleagues $(6,7)$, to assay the quality of antibodies secreted from single cells in elderly individuals compared with that in young individuals.

\section{Decreased quantity of plasmablasts contributes to decreased elderly vaccine response}

The system used by Sasaki and colleagues to analyze the antibody response to vaccination against influenza (18) enables researchers to measure primarily new antigen-specific antibody responses or those generated by switch memory B cells by focusing on plasmablasts, which peak at day 7 after exposure to a new antigen, in the case of Sasaki et al., the inactivated seasonal influenza vaccine. The plasmablasts are isolated and analyzed in bulk to assess the vaccine-specific PPAb response or, as single cells (clones), to analyze the specific Ig sequences expressed by each cell and the affinity of the mAb each cell produces. The ability to measure the vaccine-specific antibody response provides a way to avoid measuring preexisting cross-reacting serum antibody specificities generated by previous vaccinations. The system used by Sasaki 
and collaborators (18) therefore enabled them to focus on whether each vaccinated individual had the ability to generate a new beneficial antibody response or not.

Using this approach, Sasaki and colleagues were able to demonstrate that not only is the number of vaccine-specific plasmablasts decreased in elderly individuals, as compared with that in healthy young adults, but that the concentration of PPAbs is also decreased (Figure 1 and ref. 18). Conversely, the avidity of these vaccine-specific antibodies and the affinity of recombinant mAbs obtained from single cell plasmablasts were similar in the two age groups. These results support the conclusion that the lower efficacy of the influenza vaccine in the elderly is due to a quantitative difference in the number of antibody-secreting plasmablasts.

The results from Sasaki et al. (18) clearly show that the frequency of vaccine-specific plasmablasts circulating one week after vaccination is lower in elderly individuals as compared with that in young individuals. The age-dependent defect in $\operatorname{CSR}(15,16)$ (discussed earlier in Influenza infection and vaccination) leads to reduced amounts of not only IgG antibodies but also switch memory B cells (17), and this could be part of the mechanism by which vaccine-specific plasmablast numbers wane with age. The lower plasmablast response observed by Sasaki et al. in the elderly (18) may also be due to impaired reactivation of memory B cells and/ or diminished activation of naive $\mathrm{B}$ cells, the cell numbers of which have both been shown to be reduced in the elderly $(16,17,19,20)$. The vaccine-specific plasmablasts characterized by Sasaki et al. are generated in vivo, and the defect seen could also reflect deficits of age in various immune cell types other than B cells, including T cells and dendritic cells.

The work of Sasaki and collaborators shows that the amount of vaccine-specific PPAb (both IgG and IgA) secreted by bulk plasmablast cultures was decreased in elderly individuals compared with that in young individuals (18). Further, in both age groups, the amount of vaccine-specific IgG produced was statistically higher than the amount of vaccine-specific IgA. Importantly, the amount of antibody secreted by individual plasmablasts was about the same for cells isolated from both elderly and young individuals. Therefore, the primary defect in elderly individuals is in the number of plasmablasts induced by vaccination, rather than in the amount of antibody secreted per cell. Sasaki et al. also evaluated the avidity of vaccine-specific PPAbs in young and elderly individuals and found no statistically significant difference in the avidity of vaccine-specific IgG and IgA in the two age groups (18). The variable regions of the Igs isolated from single sorted plasmablasts showed no significant affinity difference, at least in the limited number of young and elderly individuals. Therefore, Sasaki and collaborators conclude that the quality of the antibodies produced (both polyclonal and monoclonal) in young and elderly individuals after influenza vaccination is comparable (18).

\section{Looking toward the future}

The work of Sasaki and colleagues (18) answers/addresses a major mechanistic question about what the defect in the response to vaccination against influenza is in elderly individuals. Importantly, it also offers a method to be able to measure and perhaps predict the quality of the vaccine-induced antibody response in all individuals vaccinated against influenza - simply analyze the frequency of the peripheral vaccine-specific plasmablast response. A decreased plasmablast response for one vaccine is likely to correlate with the response to subsequent vaccinations and so would help predict the general immune capability of the individual. It is and will be important to accurately monitor as well as predict, via the generation of accurate biomarkers, immune responses that correlate with effective disease prevention, and the work of Sasaki et al. (18) adds an important biomarker for this goal. The development of accurate biomarkers will help determine in which patients altered/enhanced vaccines should be used and beckons us to generate not only better adjuvants but also to screen for pharmaceutical and/or lifestyle changes that can improve vaccine responses to ensure effective disease prevention.

\section{Acknowledgments}

We are thankful for the support of NIH grants AG-23717 and AG-32576.

Address correspondence to: Bonnie Blomberg, University of Miami Miller School of Medicine, 3146A Rosenstiel Medical Science Building, 1600 NW 10th Avenue, Miami, Florida 33136, USA. Phone: 305.243.6040; Fax: 305.243.4623; E-mail: Bblomber@med.miami.edu.
1. Fiore AE, et al. Prevention and control of influenza with vaccines: recommendations of the Advisory Committee on Immunization Practices (ACIP), 2010. MMWR Recomm Rep. 2010; 59(RR-8):1-62

2. Simonsen L, Taylor RJ, Viboud C, Miller MA, Jackson LA. Mortality benefits of influenza vaccination in elderly people: an ongoing controversy. Lancet Infect Dis. 2007;7(10):658-666.

3. Thompson WW, et al. Influenza-associated hospitalizations in the United States. JAMA. 2004;292(11):1333-1340.

4. Brokstad KA, Cox RJ, Olofsson J, Jonsson R, Haaheim LR. Parenteral influenza vaccination induces a rapid systemic and local immune response. J Infect Dis. 1995;171(1):198-203.

5. Gerhard W. The role of the antibody response in influenza virus infection. Curr Top Microbiol Immunol. 2001;260:171-190.

6. Wrammert J, et al. Rapid cloning of high-affinity human monoclonal antibodies against influenza virus. Nature. 2008;453(7195):667-671.

7. Wrammert J, et al. Broadly cross-reactive antibodies dominate the human B cell response against 2009 pandemic H1N1 influenza virus infection. J Exp Med. 2011;208(1):181-93.

8. Radbruch A, et al. Competence and competition: the challenge of becoming a long-lived plasma cell. Nat Rev Immunol. 2006;6(10):741-750.

9. de Bruijn IA, et al. Quality and quantity of the humoral immune response in healthy elderly and young subjects after annually repeated influenza vaccination. J Infect Dis. 1999;179(1):31-36.

10. Goodwin K, Viboud C, Simonsen L. Antibody response to influenza vaccination in the elderly: a quantitative review. Vaccine. 2006;24(8):1159-1169.

11. McElhaney JE. Influenza vaccination in the elderly: seeking new correlates of protection and improved vaccines. Aging health. 2008;4(6):603-613.

12. Goronzy JJ, Fulbright JW, Crowson CS, Poland GA, O'Fallon WM, Weyand CM. Value of immunological markers in predicting responsiveness to influenza vaccination in elderly individuals. J Virol. 2001;75(24):12182-12187.

13. Pawelec G, et al. T cells and aging, January 2002 update. Front Biosci. 2002;7:d1056-d1183

14. Saurwein-Teissl M, et al. Lack of antibody production following immunization in old age: association with CD8(+)CD28(-) T cell clonal expansions and an imbalance in the production of Th1 and Th2 cytokines. J Immunol. 2002;168(11):5893-5899.

15. Frasca D, et al. Aging down-regulates the transcription factor E2A, activation-induced cytidine deaminase, and Ig class switch in human B cells. J Immunol. 2008;180(8):5283-5290.

16. Frasca D, Landin AM, Riley RL, Blomberg BB. Mechanisms for decreased function of $B$ cells in aged mice and humans. J Immunol. 2008;180(5):2741-2746.

17. Frasca D, et al. Intrinsic defects in B cell response to seasonal influenza vaccination in elderly humans. Vaccine. 2010;28(51):8077-8084.

18. Sasaki S, et al. Limited efficacy of inactivated influenza vaccine in elderly individuals is associated with decreased production of vaccine-specific antibodies. J Clin Invest. 2011;121(8):3109-3119.

19. Chong Y, et al. CD27(+) (memory) B cell decrease and apoptosis-resistant CD27(-) (naive) B cell increase in aged humans: implications for agerelated peripheral B cell developmental disturbances. Int Immunol. 2005;17(4):383-390.

20. Shi Y, Yamazaki T, Okubo Y, Uehara Y, Sugane K, Agematsu K. Regulation of aged humoral immune defense against pneumococcal bacteria by $\operatorname{IgM}$ memory B cell. J Immunol. 2005;175(5):3262-3267. 